\title{
Spatial and temporal variations in Titan's surface temperatures from Cassini CIRS observations
}

\author{
V. Cottini ${ }^{\mathrm{a}, \mathrm{b}, *}$, C.A. Nixon ${ }^{\mathrm{a}, \mathrm{b}}$, D.E. Jennings ${ }^{\mathrm{b}}$, R. de Kok $^{\mathrm{c}}$, N.A. Teanby ${ }^{\mathrm{d}}$, P.G.J. Irwin ${ }^{\mathrm{d}}$, F.M. Flasar ${ }^{\mathrm{b}}$ \\ a Department of Astronomy, University of Maryland at College Park, College Park, MD 20742, USA \\ ${ }^{\mathrm{b}}$ NASA Goddard Space Flight Center, Solar System Exploration Division, Planetary Systems Laboratory—Code 693, 8800 Greenbelt Rd, Greenbelt, MD 20771, USA \\ ' SRON, Sorbonnelaan 2, 3584 CA Utrecht, The Netherlands \\ ${ }^{\mathrm{d}}$ Atmospheric, Oceanic and Planetary Physics, University of Oxford, Oxford OX1 3PU, UK
}

\section{A R T I C L E I N F O}

\section{Article history:}

Received 10 September 2010

Received in revised form

9 March 2011

Accepted 24 March 2011

Available online 16 April 2011

\section{Keywords:}

Titan

Surface

Temperature

Infrared observations

\begin{abstract}
A B S T R A C T
We report a wide-ranging study of Titan's surface temperatures by analysis of the Moon's outgoing radiance through a spectral window in the thermal infrared at $19 \mu \mathrm{m}\left(530 \mathrm{~cm}^{-1}\right)$ characterized by lower atmospheric opacity. We begin by modeling Cassini Composite Infrared Spectrometer (CIRS) far infrared spectra collected in the period 2004-2010, using a radiative transfer forward model combined with a non-linear optimal estimation inversion method. At low-latitudes, we agree with the HASI nearsurface temperature of about $94 \mathrm{~K}$ at $10^{\circ} \mathrm{S}$ (Fulchignoni et al., 2005). We find a systematic decrease from the equator toward the poles, hemispherically asymmetric, of $\sim 1 \mathrm{~K}$ at $60^{\circ}$ south and $\sim 3 \mathrm{~K} \mathrm{at} 60^{\circ}$ north, in general agreement with a previous analysis of CIRS data (Jennings et al., 2009), and with Voyager results from the previous northern winter. Subdividing the available database, corresponding to about one Titan season, into 3 consecutive periods, small seasonal changes of up to $2 \mathrm{~K}$ at $60^{\circ} \mathrm{N}$ became noticeable in the results. In addition, clear evidence of diurnal variations of the surface temperatures near the equator are observed for the first time: we find a trend of slowly increasing temperature from the morning to the early afternoon and a faster decrease during the night. The diurnal change is $\sim 1.5 \mathrm{~K}$, in agreement with model predictions for a surface with a thermal inertia between 300 and $600 \mathrm{~J} \mathrm{~m}^{-2} \mathrm{~s}^{-1 / 2} \mathrm{~K}^{-1}$. These results provide important constraints on coupled surface-atmosphere models of Titan's meteorology and atmospheric dynamic.
\end{abstract}

(c) 2011 Elsevier Ltd. All rights reserved.

\section{Introduction}

Planetary surfaces are sources of radiation observed in the thermal infrared spectral range and their temperature changes include interactions between the surface and the atmosphere. Surface temperatures derive in part from insolation, but are also related to the exchange of heat and volatiles between the surface and atmosphere. For these reasons the surface temperature has an important role in understanding both planetary atmospheres and surface properties. At $19 \mu \mathrm{m}$, Titan's atmospheric opacity due to the gases and the thick haze layers that surround the Moon presents a minimum and the emerging radiation field has $70-80 \%$ of its contribution from the surface (Courtin et al., 1995; Flasar et al., 1981; Hanel et al., 1981; Samuelson et al., 1981). Therefore we exploit this wavelength to study the surface temperature.

\footnotetext{
${ }^{*}$ Corresponding author at: NASA Goddard Space Flight Center, Solar System Exploration Division, Planetary Systems Laboratory-Code 693, 8800 Greenbelt Rd, Greenbelt, MD 20771, USA. Tel.: +1 301286 7932; fax: +1 3012860212.

E-mail addresses: valeria.cottini@nasa.gov,

valeria.cottini@gmail.com (V. Cottini).
}

In this infrared window Voyager IRIS observed in 1980 an equator-to-pole gradient of surface brightness temperature during the northern spring of about $2-3 \mathrm{~K}$, and this remained the only information regarding Titan's surface temperatures before the Cassini mission began to observe the Moon in the following northern winter, since July 2004. In January 2005 the Huygens Atmospheric Structure Instrument (HASI) on the Huygens descent probe measured the near surface temperature (Fulchignoni et al., 2005) at its landing site $\left(10^{\circ} \mathrm{S}\right)$, assigning it a value of $93.65 \pm 0.25 \mathrm{~K}$.

During Cassini's first five years of observations of Saturn and its satellites the Composite Infrared Spectrometer (CIRS; Flasar et al., 2004) has measured the radiation coming from a large part of Titan and the latitudinal variation of surface brightness temperature has been measured from CIRS spectra using the $19 \mu \mathrm{m}$ window (Jennings et al., 2009). The inferred values are consistent with the HASI measurement near the equator and decrease asymmetrically towards the poles, being colder in the northern winter hemisphere.

With an increased number of CIRS observations of Titan we are now able to cover a larger range of longitude and different local 
conditions; this data together with the use of a radiative transfer model of the atmosphere to fit the spectra gives us an opportunity to improve our knowledge of Titan's surface temperature.

The format of the paper is as follows: in Section 2 there is a description of the type of data selected to perform this work. In Section 3 we describe the method we used to achieve the results and the model of Titan's surface and atmosphere used in the computations to simulate the observations. In Sections 4 and 5 we finally discuss the results of this study and the conclusions, respectively.

\section{Data}

Since July 2004 the Composite Infrared Spectrometer (CIRS) on board the Cassini mission to the Saturnian system has been acquiring spectra of Titan.

For the task of surface temperature retrieval we analyze spectra detected by the CIRS Focal Plane 1 detector (FP1) with a spectral resolution of $15.5 \mathrm{~cm}^{-1}$ and a field of view of $3.9 \mathrm{mrad}$. The FP1 detector acquires data in the far infrared $\left(10-600 \mathrm{~cm}^{-1}\right)$; we study the range $350-530 \mathrm{~cm}^{-1}$ in order to cover the spectral region with the lowest atmospheric opacity, which allows us to penetrate the thick layer of haze and gases and sense the surface. Despite the fact that up to $600 \mathrm{~cm}^{-1}$ the atmospheric opacity is still low we use the spectrum only below $530 \mathrm{~cm}^{-1}$ to avoid instrumental electrical interferences sometimes present at higher wavenumbers, as well as higher detector noise. We also did not attempt to fit the range below $350 \mathrm{~cm}^{-1}$ where the influence of the poorly known tropospheric temperature profile becomes increasingly important.

We initially used data coming from 2 different types of observations: the FIRNADMAP (Far Infrared Nadir Map) and FIRNADCMP (Far Infrared Nadir Composition Integrations). The FIRNADMAPs are slow slews usually executed from north to south or east to west across the visible part of the disk at low spectral resolution $15.5 \mathrm{~cm}^{-1}$, intended specifically for surface temperature mapping. In this category we also include similar observations performed by the UVIS instrument, known as EUVFUV maps where CIRS is acquiring data in 'ride-along' mode. FIRNADCMP sequences are designed to measure the abundances of trace gases in Titan's stratosphere from far-infrared emissions. Rather than continuous slewing for mapping, they are instead characterized by long integration times and a higher spectral resolution of $0.5 \mathrm{~cm}^{-1}$, degraded to $15.5 \mathrm{~cm}^{-1}$ to increase signal-to-noise for the purpose of our study. These observations build up a spatial map of the trace gases across the disk from multiple flybys, observing only one location at a time.

We treated the two databases in two different ways, due to the different distances from which the probe was observing Titan, which determines a different footprint size on the surface. The FIRNADMAP sequences are typically acquired from distances less than $100,000 \mathrm{~km}$, at which range the FP1 field of view (FOV) of 3.9 mrad has a sub-spacecraft (minimum) footprint of $200-300 \mathrm{~km}$ on the surface. The FIRNADCMP long-integration sequences are normally executed from greater distances, at ranges 160,000 to $270,000 \mathrm{~km}$, yielding minimum footprints of 800-1000 km.

From an initial set of about 127,000 nadir spectra acquired by CIRS-FP1 and already selected to have the entire footprint on Titan's disk, we then chose those with (i) the entire FOV on the Moon and (ii) with an emission angle at the footprint center less than $70^{\circ}$ (most of the data acquired from an angle smaller than $50^{\circ}$ ) and (iii) acquired from a distance less than $180,000 \mathrm{~km}$ for the FIRNADMAP sequences and less than $270,000 \mathrm{~km}$ for the
FIRNADCMP. These selections bring the number of the spectra considered to about 70,000.

We also restricted our selection to consecutive observations in which the movement in mrad of the center of the footprint during the spectral scan is smaller than $0.1 \%$ of the field (less than $0.02 \%$ for most of the data). These criteria further reduced the amount of data under consideration but allowed us to obtain a more localized FOV on the surface. The noise in this spectral range being quite high, we averaged data in groups of about 60 close observations to decrease the noise background in order to be able to study the continuum level. These small block averages were constructed from consecutive observations, using the constraints described above, which allowed us to consider averages of radiance coming from the surface and atmosphere with the same physical conditions and acquired from similar distances and angles.

Besides these selections, to avoid biased results for possible seasonal changes of the surface temperature, we also subdivided our database, from December 2004 to July 2010, into 3 different periods of data acquisition. A list of the periods and the corresponding observations can be found in Table 1 and Fig. 1.

Due to the orbit followed by the probe around Titan, in December 2004 the sub-spacecraft point was positioned just below the equator. Therefore during the first time period considered the emission angles of the FIRNADMAP observations increased towards the north and south poles with latitude, so that higher latitudes were observed at higher emission angles, while for the second and third period smaller emission angles were also possible for higher latitude observations. While the FIRNADMAP sequences usually observed the surface along a N-S line, resulting in low emission angles at low latitudes, the FIRNADCMP observations were typically performed with medium or high emission angles at all latitudes, with many data having emission angles between $45^{\circ}$ and $60^{\circ}$. Due to modeling uncertainties caused by the large, inhomogeneous field of view at high latitudes, we finally used only the FIRNADCMP results acquired with lower emission angles, which in these data corresponds to the equatorial region, and mainly as a confirmation of the results obtained by the FIRNADMAP database.

The FOV projected on the surface of the observations used in this paper, given the maximum values of spacecraft distance and emission angle considered, ranges from 100 to $900 \mathrm{~km}$, but most of the data have a footprint of 300 or $500 \mathrm{~km}$. Considering that $1^{\circ}$ of arc of a great circle corresponds to $\sim 45 \mathrm{~km}$, we can see that most of the results shown in Section 4 have a projected footprint on the surface corresponding to a value between $5^{\circ}$ and $10^{\circ}$ of latitude.

\section{Method and model}

In order to obtain information on the surface temperature from the FP1 spectral continuum we applied a quantitative model developed at Oxford University, NEMESIS (Irwin et al., 2008), to compute the spectral radiances for comparison with the measurements.

For this purpose we used a combination of forward model and retrieval scheme based on the method of optimal estimation (Rodgers, 1976, 2000). The forward model calculates Titan's emerging radiation field using precomputed absorption coefficients in a correlated- $k$ model (Lacis and Oinas, 1991) for the computations of atmospheric opacity. The gaseous bands and collision-induced absorption (CIA) opacities $k$-coefficients between nitrogen, methane and hydrogen molecules (therefore six collision pairs in total) are pretabulated over the range of pressures and temperatures required to model the Titan atmosphere. The spectral 
Table 1

CIRS and UVIS-led far-infrared nadir map observations considered for our study. The latitude, longitudes, local times and projected footprint ranges of each observation are

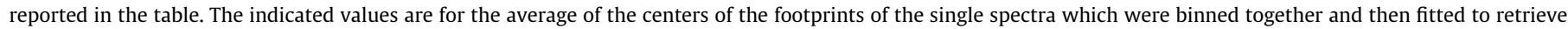
the surface temperature. The number of data is the number of considered average in each observation.

\begin{tabular}{|c|c|c|c|c|c|c|}
\hline & Latitude (N) & Longitude (W) & Time (h) & Footprint (km) & Date & N. of data \\
\hline \multicolumn{7}{|l|}{ Observation name (Period 1) } \\
\hline OOBTI_FIRNADMAP001_UVIS & $-55-+49$ & 115-184 & 10-14 & $458-840$ & 14 December 2004 & 35 \\
\hline OOBTI_FIRNADMAP002_UVIS & $+22-+59$ & 319-339 339.1 & 0-2 & 194-495 & 14 December 2004 & 14 \\
\hline 006TI_FIRNADMAP003_UVIS & $-58-+56$ & 27-30 & 15 & $407-820$ & 16 April 2005 & 22 \\
\hline 013TI_FIRNADMAP002_PRIME & $-53-+54$ & 24-27 & 15 & $280-480$ & 22 August 2005 & 19 \\
\hline 013TI_FIRNADMAP003_PRIME & $-11--5$ & $154-274$ & $23-7$ & 266-509 & 22 August 2005 & 18 \\
\hline 019TI_FIRNADMAP005_UVIS & $-\mathbf{5 0}-+\mathbf{5 1}$ & 198-202 & 2 & $452-836$ & 26 December 2005 & 13 \\
\hline 021TI_FIRNADMAP003_UVIS & $-57-+30$ & 199-224 & $22-3$ & 535-906 & 27 February 2006 & 26 \\
\hline 023TI_FIRNADMAP003_UVIS & $-24-+7$ & $192-258$ & 18-23 & $596-886$ & 1 May 2006 & 15 \\
\hline 024TI_FIRNADMAP002_UVIS & $-13-+15$ & 97-213 & $2-10$ & 431-698 & 20 May 2006 & 23 \\
\hline 024TI_FIRNADMAP003_PRIME & $-50-+39$ & 341-344 & 17 & 263-399 & 20 May 2006 & 9 \\
\hline 025TI_FIRNADMAP003_UVIS & $-12-+19$ & 139-256 & 16-24 & 333-855 & 2 July 2006 & 22 \\
\hline 026TI_FIRNADMAP003_PRIME & $-52-+35$ & 338-339 & 16 & 271-421 & 22 July 2006 & 8 \\
\hline 028TI_FIRNADMAP002_UVIS & $-1-+16$ & 104-198 & $2-7$ & 412-728 & 7 September 2006 & 10 \\
\hline 029TI_FIRNADMAP002_UVIS & $\mathbf{0}-+\mathbf{1 7}$ & 90-192 & $1-8$ & $400-729$ & 23 September 2006 & 12 \\
\hline \multicolumn{7}{|l|}{ Observation name (Period 2) } \\
\hline 035TI_EUVFUV001_UVIS & $+14-+32$ & 72-186 & 1-9 & $372-540$ & 12 December 2006 & 13 \\
\hline 036TI_FIRNADMAP002_PRIME & $-22-+58$ & 132-133 & 5 & $294-454$ & 28 December 2006 & 18 \\
\hline 036TI_FIRNADMAP003_PRIME & $-59-+3$ & 318-319 & 17 & 311-415 & 28 December 2006 & 9 \\
\hline 038TI_EUVFUV001_UVIS & $+30-+53$ & 58-174 & 2-9 & $523-859$ & 28 January 2007 & 10 \\
\hline 040TI_EUVFUV002_UVIS & $-10-+53$ & 211-237 & 10-12 & $476-838$ & 9 March 2007 & 12 \\
\hline 040TI_FIRNADMAP001_PRIME & $-56-+5$ & 43 & 22 & 284-452 & 10 March 2007 & 10 \\
\hline 040TI_FIRNADMAP002_PRIME & +58 & 209 & 12 & 333 & 10 March 2007 & 1 \\
\hline 041TI_EUVFUV001_UVIS & $-59--55$ & 45-52 & 22 & 220 & 25 March 2007 & 2 \\
\hline 041TI_EUVFUV002_UVIS & $-7-+57$ & 212-235 & 10-12 & $458-747$ & 26 March 2007 & 11 \\
\hline 041TI_FIRNADMAP002_PRIME & $+\mathbf{5 1}$ & 223 & 11 & 220 & 26 March 2007 & 1 \\
\hline 043TI_FIRNADMAP001_PRIME & $-56-+30$ & 28 & 23 & 283-672 & 26 April 2007 & 15 \\
\hline 044TI_EUVFUV001_UVIS & +5 & 25 & 23 & 434 & 12 May 2007 & 2 \\
\hline 045TI_EUVFUV001_UVIS & $-51-+45$ & 19-22 & 23 & $555-922$ & 28 May 2007 & 26 \\
\hline 046TI_FIRNADMAP001_ISS & o & 25 & 23 & 170-216 & 13 June 2007 & 4 \\
\hline 046TI_FIRNADMAP003_VIMS & +15 & 220 & 11 & 459-530 & 13 June 2007 & 11 \\
\hline 046TI_FIRNADMAP902_PRIME & $-\mathbf{5 5}-+\mathbf{5 0}$ & 210 & 11 & $278-496$ & 13 June 2007 & 21 \\
\hline 046TI_FIRNADMAP902_VIMS & +58 & 232 & 10 & 168 & 13 June 2007 & 1 \\
\hline 047TI_FIRNADMAP002_PRIME & $+2-+56$ & 208 & 11 & $309-639$ & 29 June 2007 & 8 \\
\hline 048TI_EUVFUV001_UVIS & $-58-+52$ & 144-147 & 20 & $511-968$ & 19 July 2007 & 13 \\
\hline 048TI_FIRNADMAP002_PRIME & $\mathbf{0}-+10$ & $352-22$ & 4-8 & $303-589$ & 19 July 2007 & 8 \\
\hline 049TI_FIRNADMAP001_PRIME & $-47--53$ & 13-16 & 22 & 278-537 & 31 August 2007 & 10 \\
\hline 050TI_EUVFUV001_UVIS & $-27-+52$ & 10-12 & 22 & $582-810$ & 1 October 2007 & 9 \\
\hline 052TI_FIRNADMAP001_PRIME & $-56-+55$ & 19-23 & 21 & 274-665 & 18 November 2007 & 21 \\
\hline 052TI_FIRNADMAP002_PRIME & $-20-+4$ & 211 & 9 & $307-367$ & 19 November 2007 & 3 \\
\hline 053TI_FIRNADMAP001_PRIME & $-57-+50$ & 24-27 & 21 & 299-711 & 4 December 2007 & 20 \\
\hline 053TI_FIRNADMAP002_PRIME & $-40-+9$ & 161-211 & 9-13 & $312-691$ & 5 December 2007 & 8 \\
\hline 055TI_EUVFUV001_UVIS & $-35-+7$ & $346-71$ & 17-24 & 513-872 & 5 January 2008 & 41 \\
\hline 055TI_EUVFUV501_UVIS & $+1-+15$ & $336-354$ & 23-24 & $497-830$ & 5 January 2008 & 9 \\
\hline 055TI_FIRNADMAP002_PRIME & $+31-+52$ & 221-232 & 8 & $380-408$ & 6 January 2008 & 4 \\
\hline 059TI_EUVFUV002_UVIS & $+55-+59$ & 144-147 & 12-14 & $507-689$ & 22 February 2008 & 3 \\
\hline 062TI_FIRNADMAP001_PRIME & $-57-+30$ & $43-46$ & 19 & 291-705 & 26 March 2008 & 16 \\
\hline 062TI_FIRNADMAP002_PRIME & $+39-+59$ & 236 & 7 & $370-400$ & 26 March 2008 & 4 \\
\hline 067TI_FIRNADMAP002_PRIME & $-19-+58$ & 241-243 & 7 & 286-523 & 13 May 2008 & 15 \\
\hline 069TI_EUVFUV001_UVIS & $-54-+24$ & $56-59$ & 18 & $472-940$ & 28 May 2008 & 13 \\
\hline \multicolumn{7}{|l|}{ Observation name (Period 3) } \\
\hline 093TI_FIRNADMAP002_PRIME & $-29-+55$ & 253-257 & 5 & $283-561$ & 20 November 2008 & 17 \\
\hline 095TI_EUVFUV001_UVIS & $-16-+49$ & 260-271 & 4-5 & 539-834 & 6 December 2008 & 8 \\
\hline 107TI_FIRNADMAP002_PRIME & $+13-+58$ & 245 & 6 & $308-533$ & 27 March 2009 & 8 \\
\hline 108TI_FIRNADMAP002_PRIME & $-52-+2$ & 268 & 16 & $195-356$ & 4 April 2009 & 8 \\
\hline 110TI_EUVFUV001_UVIS & $-59--22$ & 204-322 & 11-20 & 512-904 & 5 May 2009 & 21 \\
\hline 110TI_FIRNADMAP001_PRIME & $+75-+81$ & 158 & 4 & $286-468$ & 5 May 2009 & 13 \\
\hline 111TI_EUVFUV001_UVIS & $+9-+60$ & 80-220 & $2-18$ & $484-850$ & 21 May 2009 & 8 \\
\hline 111TI_FIRNADMAP002_PRIME & $-55--1$ & $254-268$ & 16-17 & 296-533 & 21 May 2009 & 13 \\
\hline 112TI_EUVFUV001_UVIS & $-57--6$ & $20-340$ & 7-15 & $503-860$ & June 2009 & 11 \\
\hline 113TI_EUVFUV001_UVIS & $-12-+57$ & 120-133 & 0-2 & 492-978 & 22 June 2009 & 15 \\
\hline 113TI_EUVFUV002_UVIS & $-57--3$ & 314-331 & $12-13$ & $552-704$ & 23 June 2009 & 11 \\
\hline 114TI_EUVFUV001_UVIS & $+21-+56$ & 134 & 0 & $319-368$ & 8 July 2009 & 7 \\
\hline 114TI_FIRNADMAP002_PRIME & $-56-+26$ & 258-298 & 13-17 & 226-386 & 8 July 2009 & 13 \\
\hline 115TI_FIRNADMAP002_PRIME & $-56-+34$ & $308-344$ & 10-13 & $266-451$ & 24 July 2009 & 9 \\
\hline 119TI_EUVFUV002_UVIS & $-54-+34$ & $323-10$ & 9-13 & 519-921 & 12 October 2009 & 11 \\
\hline 123TI_FIRNADMAP001_PRIME & $-53-+58$ & 12-32 & $2-3$ & 285-672 & 27 December 2009 & 22 \\
\hline 124TI_FIRNADMAP001_PRIME & $+1-+60$ & $322-80$ & $15-7$ & $307-633$ & 12 January 2010 & 14 \\
\hline 124TI_FIRNADMAP002_PRIME & $-57--23$ & $153-271$ & 10-19 & 310-701 & 13 January 2010 & 12 \\
\hline
\end{tabular}


Table 1 (continued)

\begin{tabular}{|c|c|c|c|c|c|c|}
\hline & Latitude ( $\mathbf{N})$ & Longitude (W) & Time (h) & Footprint (km) & Date & N. of data \\
\hline 125TI_EUVFUV001_UVIS & $-3-+1$ & $320-65$ & 1-7 & 486-854 & 28 January 2010 & 14 \\
\hline 125TI_EUVFUV002_UVIS & $-8-+2$ & 157-255 & 12-19 & $517-813$ & 29 January 2010 & 11 \\
\hline 129TI_FIRNADMAPO01_PRIME & $-50-+7$ & 94-190 & $20-3$ & $336-714$ & 5 April 2010 & 19 \\
\hline 129TI_FIRNADMAP002_PRIME & $-\mathbf{2 5}-+57$ & 285-335 & 10-15 & $343-578$ & 5 April 2010 & 8 \\
\hline 132TI_EUVFUV001_UVIS & $-5-+5$ & 175-267 & 10-17 & $480-764$ & 5 June 2010 & 12 \\
\hline
\end{tabular}

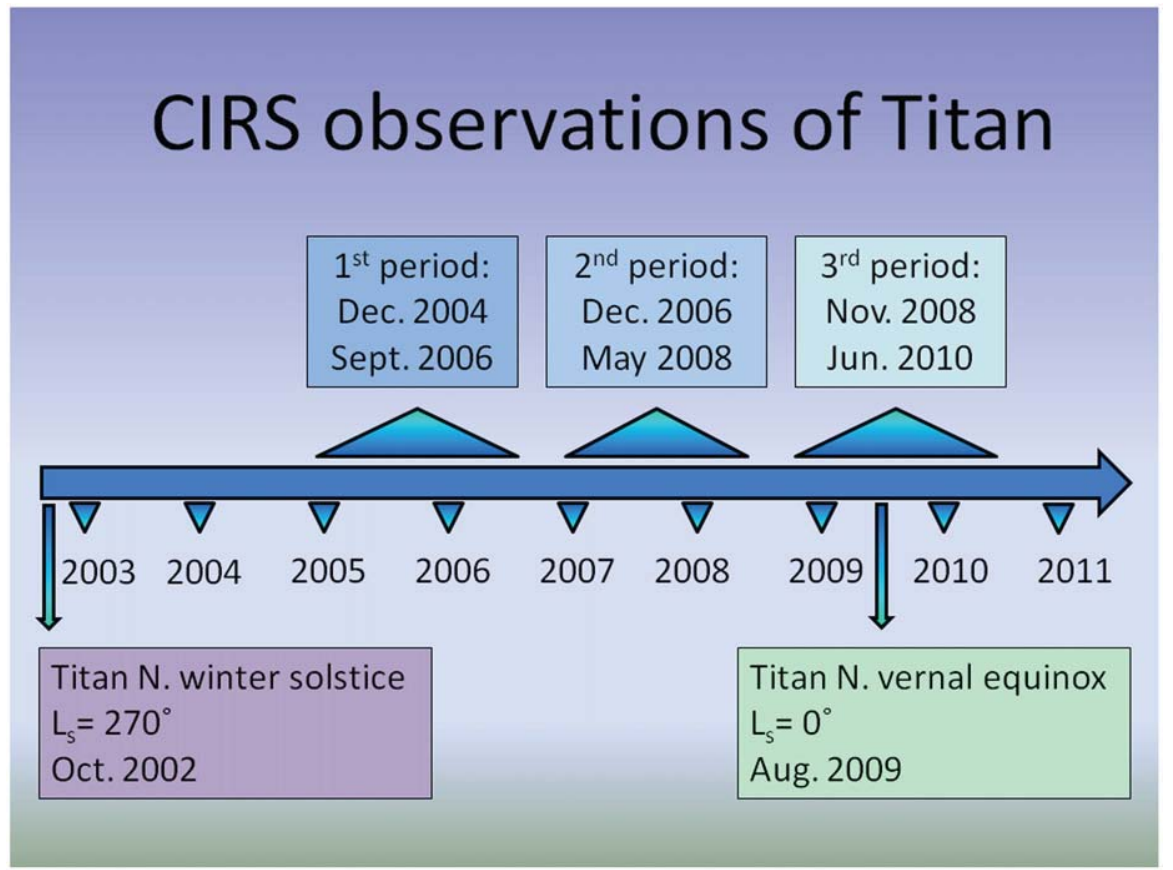

Fig. 1. Last Titan northern winter/southern summer correspondence with the terrestrial years of the Cassini mission to the Moon. The subdivision of the data in 3 periods of time (Period 1-from December 2004 to July 2006, Period 2-from September 2006 to May 2008; Period 3-from November 2008 to January 2010) is also shown. The width of the upper triangles indicates the actual length of the corresponding period: the 3 time periods have a similar length and no observations in between.

opacities are pre-convolved with a Hamming apodization function with the same resolution as the FP1 data.

The retrieval algorithm iteratively computes a synthetic spectrum, compares it to the data and then minimizes a cost function to determine the best estimates for the physical parameters in the model (surface temperature and stratospheric haze profile). The cost function includes a component which measures the quality of the fit to the spectra (similar to a $\chi^{2}$ test) and another which considers the deviation of the retrieved parameters from a set of a priori quantities. We use a priori conditions to constrain the retrieval process to search for physically realistic situations and avoid unphysical ones: regions of parameter space that might otherwise be explored due to the ill-posed nature of the retrieval problem. The radiative transfer equation, solved for 147 spherical atmospheric layers, has as source function the thermal emission of the surface and the atmospheric layers, which are the predominant contributions in the observed range of the spectrum. We include haze emission/absorption but do not include scattering in our computations since it is negligible at these wavelengths for particles smaller than few microns, as expected for Titan's stratospheric haze. We do not yet include tropospheric clouds in our model. We define an input atmospheric and surface model as follows.

In the model we adopted the atmospheric vertical temperature-pressure profile (from the surface to $7 \times 10^{-12}$ bar at $10^{\circ} \mathrm{S}, 168^{\circ} \mathrm{E}$ ) and the $\mathrm{CH}_{4}$ volume mixing ratio acquired by
Huygens probe in its descent to Titan's surface (Niemann et al. 2005; Fulchignoni et al., 2005) with values varying from 5\% close to the surface to $1.6 \%$ in the upper troposphere and $1.4 \%$ in the stratosphere (assumed uniformly mixed). Heights are calculated from hydrostatic equilibrium. It was possible to use the same methane profile for all the retrievals since it does not vary significantly with latitude in the troposphere, as shown by Penteado et al. (2010). The volume mixing ratio of $\mathrm{H}_{2}$ is assumed to have a uniform value of $0.1 \%$ (Courtin et al., 2008).

The $\mathrm{CH}_{4}$ spectroscopic information, which we used only to take into account the wings of the rotational lines in the sub-mm that may affect our range, were extracted from the HITRAN 2004 database (Rothman et al., 2005).

The most important contribution to the far-infrared opacity, which affects the level of the spectrum continuum, is given by the collision-induced absorption of the atmospheric molecules $\mathrm{N}_{2}$, $\mathrm{CH}_{4}$ and $\mathrm{H}_{2}$, calculated according to Borysow and Frommhold (1986a,b, c, 1987), Borysow (1991) and Borysow and Tang (1993). For the $\mathrm{N}_{2}-\mathrm{CH}_{4}$ pair we used CIA coefficient values increased by $50 \%$ as recommended in de Kok et al. (2010) based on a comparison between model prediction and the far-infrared continuum data.

Haze is also absorbing in the far-infrared and its density and extinction cross sections were taken from de Kok et al. (2007). Below $80 \mathrm{~km}$ altitude the haze scale height is the same as that of 

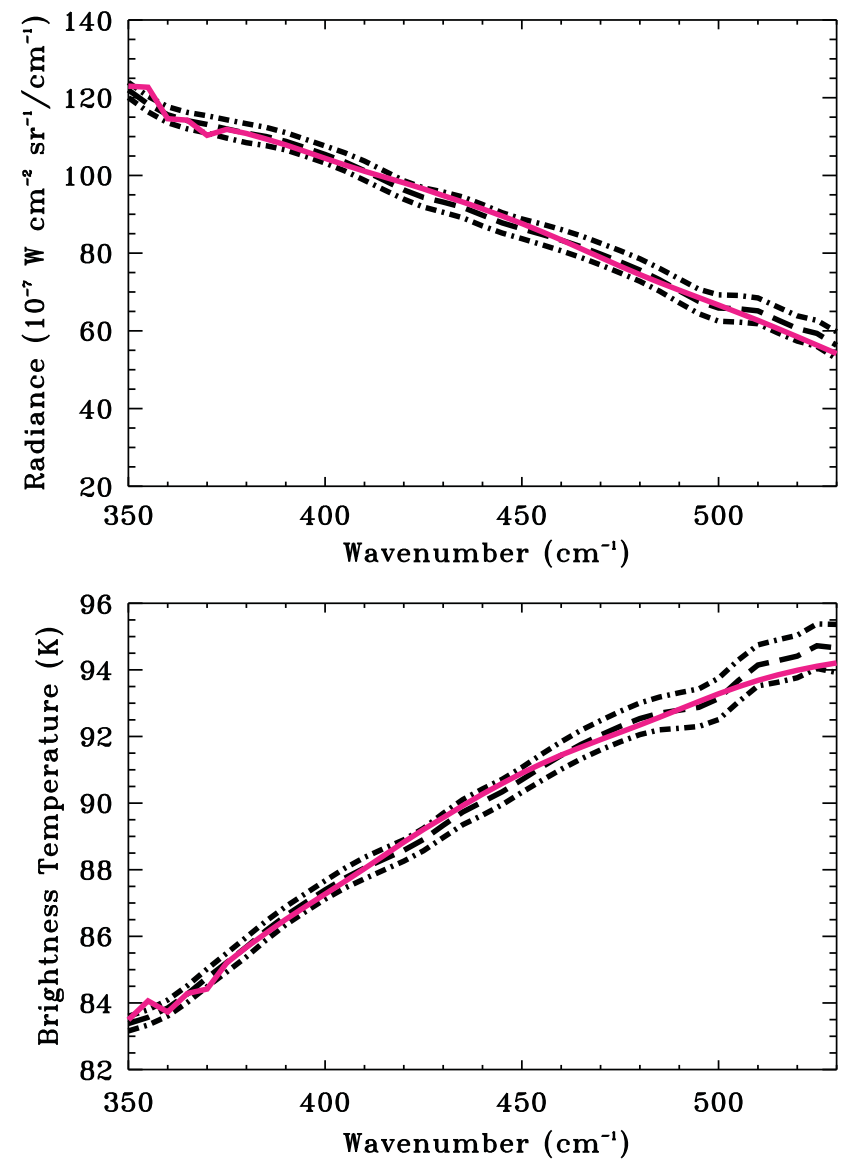

Fig. 2. Example of fitting an averaged spectrum shown in terms of radiance and brightness temperature. In dashed line is a single average of 60 spectra, plus/ minus three times their standard deviation (in dash dot). The fit is shown in solid line.

atmospheric pressure while above $80 \mathrm{~km}$ it has a scale height of $65 \mathrm{~km}$. A multiplying scale factor to this initial density profile is then retrieved together with the surface temperature values. A unit surface emissivity is assumed in the model and the observation geometry of the acquisitions is included.

In Fig. 2 we show an example of a single spectral average in the range we used for the retrieval and its fit, plus and minus 3 times the standard deviation of the 60 spectra used to build the average.

\section{Results and discussion}

The retrieved values for the Titan surface temperatures from the Cassini CIRS data show a consistency with previous studies but also add interesting new insights to the topic. In the following sections the results of the surface temperatures in relation to latitude, longitude, local time and haze will be described.

\subsection{Latitudinal and seasonal variations of surface temperature and haze}

We found for the surface temperature a value of about $94 \mathrm{~K}$ around the equator, close to the HASI result of $93.6 \mathrm{~K}$ at a latitude of $10^{\circ} \mathrm{S}$ (Fulchignoni et al., 2005) and with previous work by Jennings et al. (2009).

Fig. 3 shows an example of the surface temperature for a single north-south scan of the planet. The error bar on the single temperature retrieval is computed by the retrieval code depending on

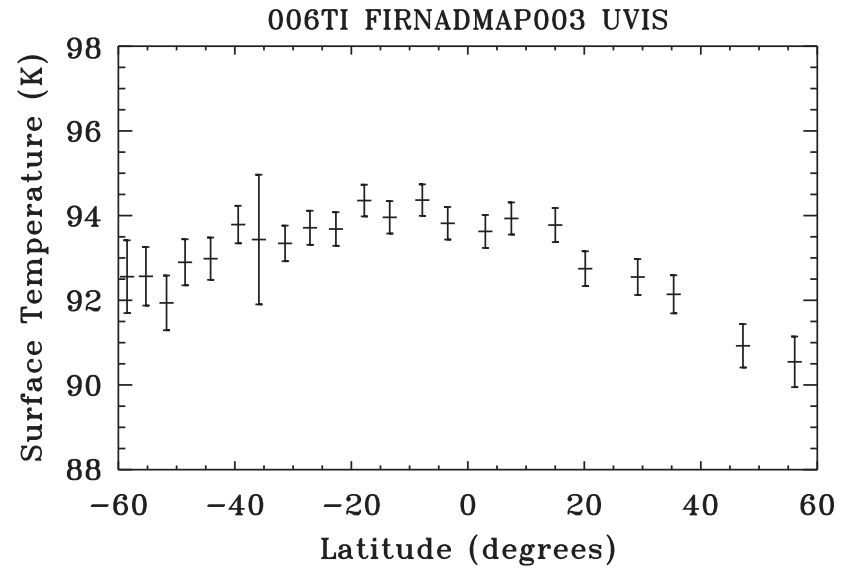

Fig. 3. Retrieved surface temperatures for a single FIRNADMAP sequence on Titan acquired on April 16, 2005. The error bar on the single temperature retrieval is computed by the retrieval code depending on the NESR of the instrument, the a priori error used in input to the retrieval algorithm in order to exclude unrealistic surface temperatures, the standard deviation of the 60 spectra averaged together to build the "single" data from which we retrieve the surface temperature values; the result is an error ranging from about 0.5 to $1 \mathrm{~K}$ for single retrievals.

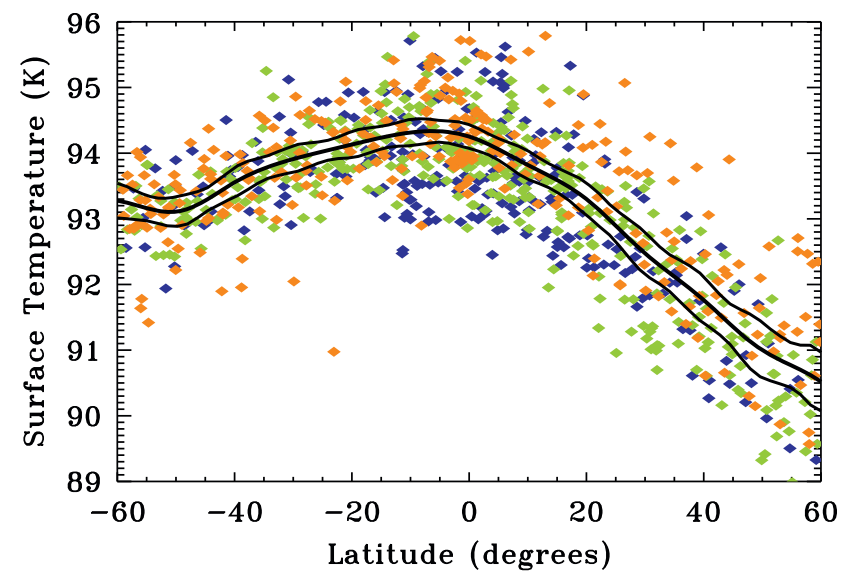

Fig. 4. Scatter plot of the individual surface temperature retrieval results as a function of latitude for the 3 time periods in which we subdivided the observation s (Period 1: December 2004-July 2006 in blue, Period 2: September 2006-May 2008 in green, Period 3: November 2008-January 2010 in orange), going from the central part of the winter to the beginning of the spring. In thick solid black is shown the smooth spline fit with its 3-sigma fit error.

the noise equivalent spectral radiance (NESR) of the instrument $\left(\sim 1.5 \mathrm{e}^{-6} \mathrm{~W} \mathrm{~cm}^{-2} \mathrm{sr}^{-1} / \mathrm{cm}^{-1}\right)$, the a priori error ( $\left.2 k\right)$ used in input to the retrieval algorithm in order to exclude unrealistic surface temperatures, the standard deviation of the 60 spectra averaged together to build the "single" data from which we retrieve the surface temperature values; the result is an error ranging from about 0.5 to $1 \mathrm{~K}$ for single retrievals.

Since we currently lack knowledge about the physical atmospheric and surface conditions at high latitudes - especially the temperature profile changes in the tropopause over $\pm 60^{\circ}$, and a model for clouds and potentially different albedo/emissivity of the lakes - we considered for this study only the results for latitudes lower than $\pm 60^{\circ}$ to properly fit and therefore reliable, which are now presented.

The surface temperature values obtained fall in the range 91.0-94.5 $\mathrm{K}$ as can be observed in Fig. 4. We have plotted the temperature results for each single average; we then used the method of fitting a smooth cubic spline curve through the data 


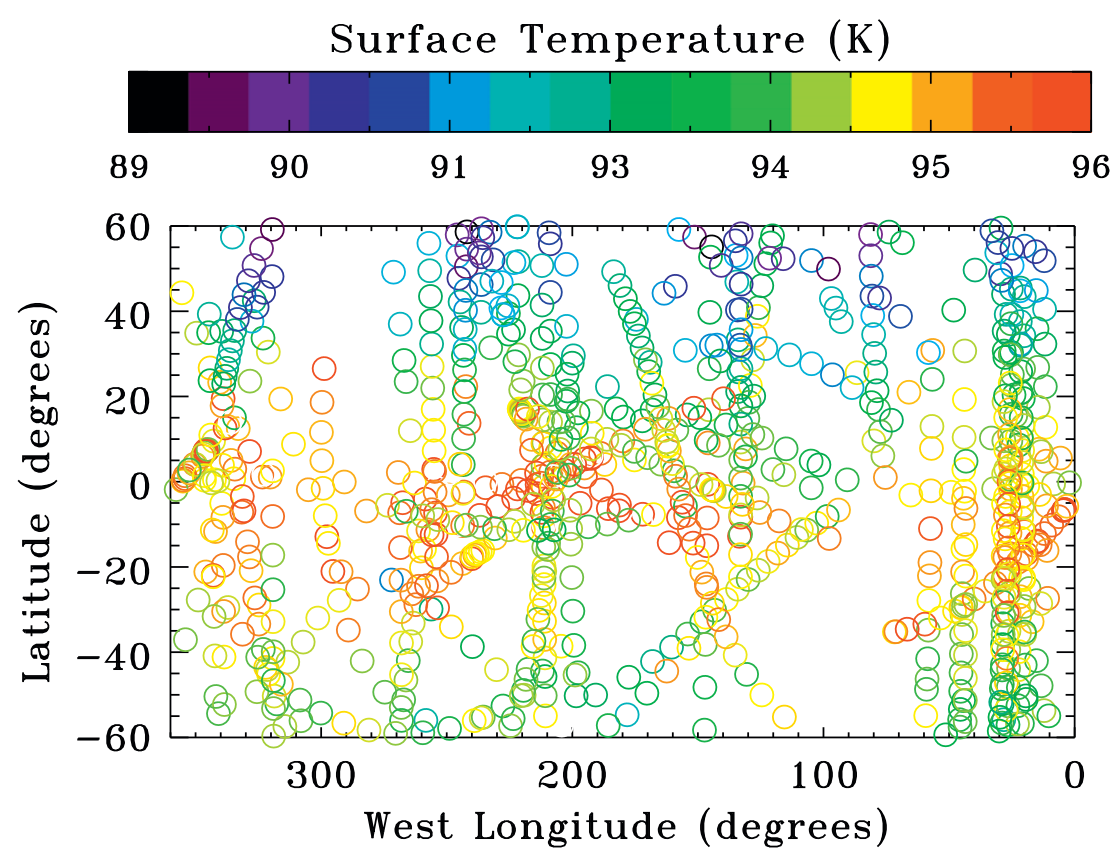

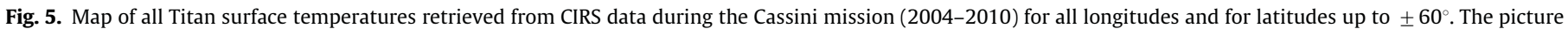

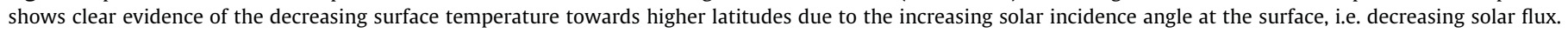

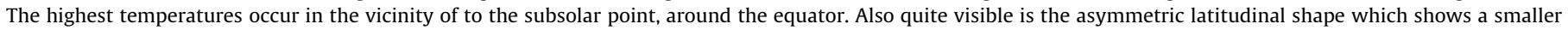

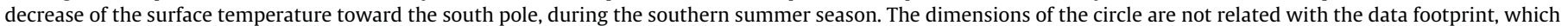

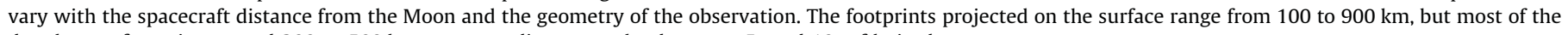
data have a footprint around 300 or $500 \mathrm{~km}$ corresponding to a value between $5^{\circ}$ and $10^{\circ}$ of latitude.

points (Teanby, 2007). This allowed us both to fit the data and to retrieve the uncertainties of the fit. These uncertainties were determined through a linear inversion process which maps the individual retrieval errors into the covariance of the fitting parameters. The overall fit errors were also scaled by $\sqrt{ } \chi^{2} / n$ to account for additional scatter in the retrieval results.

We can clearly observe the dependence of the surface temperature on the latitude with an evident maximum $10-15^{\circ}$ below the equator and a decrease towards the poles of about $1 \mathrm{~K}$ toward the south pole and $3 \mathrm{~K}$ toward the north pole. Showing the single results also gives us the chance to observe the scatter of all the data, which actually comprises both the random error and other variations of the surface temperature, including parameters other than the latitude.

The lower values reached in the northern hemisphere derive from the Titan season: the Cassini flyby right after the northern winter solstice was the epoch in which CIRS started to acquire Titan spectra, while the most recent acquisitions occurred in the early northern spring. In units of a Titan year ( $\sim 29.5$ Earth years) the Cassini mission has now observed almost one entire season (see Fig. 1), from a third of season after the northern winter solstice occurred in October 2002 and the northern spring equinox, in August 2009.

In Fig. 5 we present the map of Titan surface temperature for all longitudes and for latitudes smaller than $60^{\circ}$. The picture shows evidence of decreasing surface temperature towards higher latitudes, due to the increasing solar incidence angle at the surface, i.e. decreasing solar flux. The highest temperatures occur in the vicinity of the subsolar point, around the equator. The asymmetric latitudinal shape is also visible, which shows a smaller decrease of surface temperature toward the south pole, during the southern summer season.

Besides representing all the available results together we also divided them in the three time periods described in Section 2 in order to look for changes over time (seasonal variation).
We also separated the day time and night time data (Fig. 6a and $b$ ). The day and night have been defined considering the Sun incidence angle at the observed location on the surface, in order to take into account the changes in local time of the beginning and end of the day for different latitudes and seasons. This further subdivision was introduced in order to exclude confusion of seasonal effects with biases due to diurnal variations, also investigated and presented later in Section 4.2.

In Fig. 6 the temperature results are averaged in bins of ten degrees of latitude to better show the general surface temperature trend; the error bar is estimated as 3 times the standard deviation (using the above described errors as weights) of the averaged data in bins of latitude.

Fig. 4 shows instead the single surface temperature results, with different color for the 3 periods of the season, so the reader can have a clearer comprehension on how the data are distributed in latitude over time.

Some changes of temperature towards the north pole are noticeable among the three periods which span almost the whole winter. The first and second database analyzed (Fig. 6), which corresponds to the central part of the northern winter, show lower values toward the north pole compared to the third period corresponding to the end of the winter/early spring, which instead shows higher northern temperatures. The effect appears more evident in the night results, possibly because fewer data acquired in the third period during the day are available to be compared with the earlier winter ones (in Fig. 6a data for the last time period in the north are missing). At $60^{\circ}$ north the difference of the surface temperature between the winter data and the early spring ones reaches a value of about $2 \mathrm{~K}$. Much smaller seasonal differences, and characterized by a trend inversion, appear in the southern hemisphere up to $60^{\circ}$ of latitude, except that most of the surface temperatures observed during the day are higher than the ones observed during the night. 
a

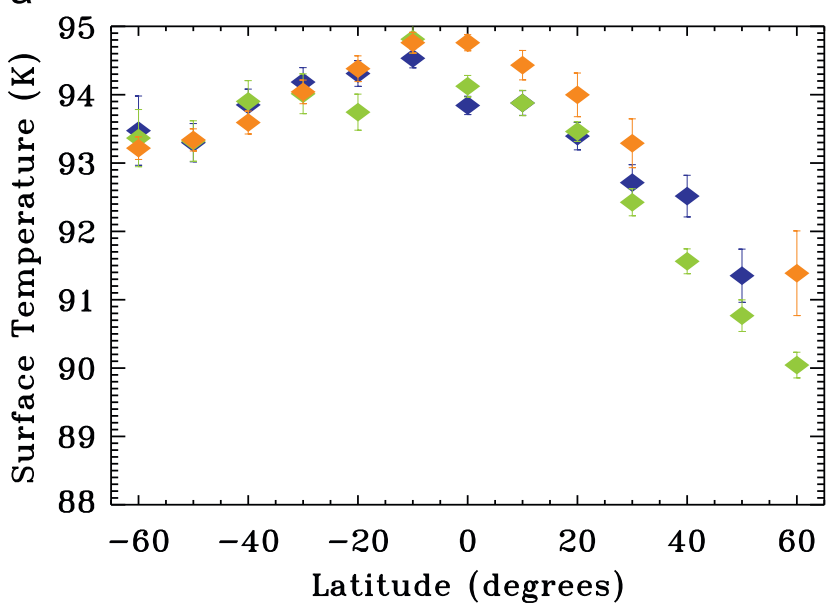

b

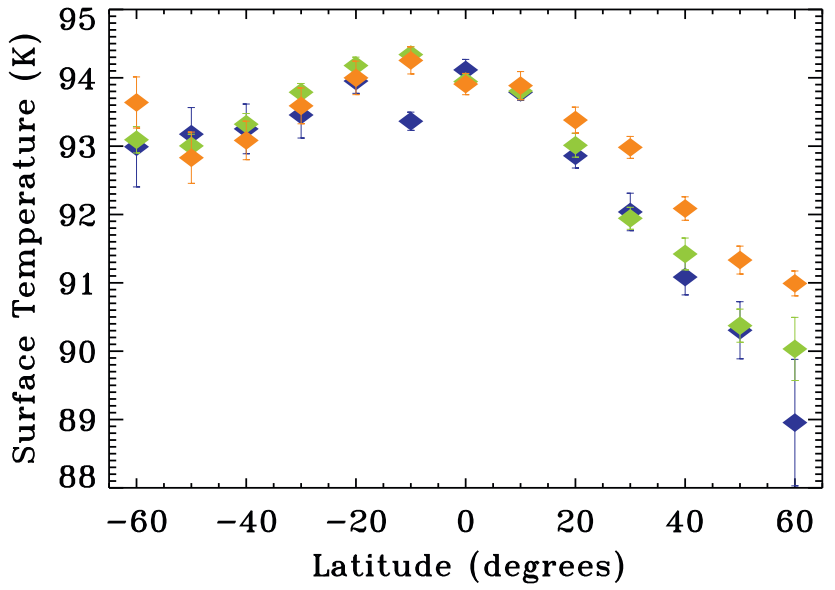

Fig. 6. Seasonal surface temperature variations for day side data (a) and night side data (b). The surface temperature results as a function of latitude are subdivided into different periods and are averaged in bins of ten degrees of latitude. The periods, three consecutive divisions of the data acquired by CIRS during the Titan flybys (northern middle of the winter to the beginning of the spring) are: Period 1 -middle of the northern winter, data acquired from December 2004 to July 2006 (blue); Period 2-late northern winter, data acquired from September 2006 to May 2008 (green); Period 3-beginning of the northern spring, data acquired from November 2008 to January 2010 (orange). Changes of temperature in the northern hemisphere are observed going toward the north pole: the central part of the northern winter presents lower temperature values than the early spring.

We expect that future analyses will show these trends more strongly, with the addition of further maps from the continuing Cassini Solstice Mission and possibly more information on the atmospheric characteristics of the high latitudes, allowing us to investigate latitudes higher than $60^{\circ}$, where the seasonal variations may be more evident.

A multiplying factor to the input haze profile has been retrieved at the same time as the surface temperature. While the spectral range fitted is more sensitive to the surface temperature at wavenumbers near $500 \mathrm{~cm}^{-1}$ and to tropospheric temperature at lower wavenumbers (toward $300 \mathrm{~cm}^{-1}$ ), the haze contribution in this relatively small spectral range can be considered almost constant in wavenumber. Although the haze opacities likely do vary with latitude and have a different influence in the spectrum, the retrieved variations may include an ambiguity between real haze variations and possible variations in atmospheric temperature, which have been ignored by assuming the HASI low-latitude profile at all latitudes. Other uncertainties may result from radiance calibration inaccuracies.

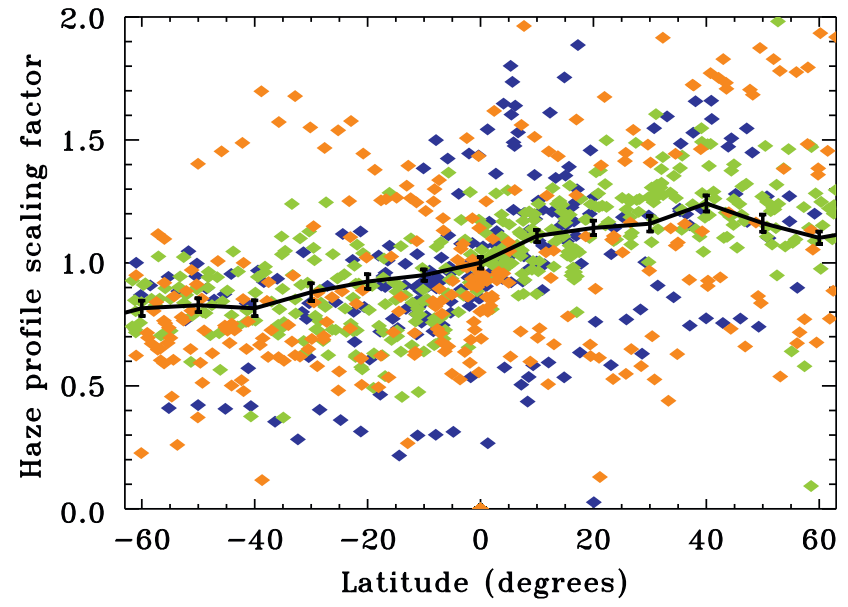

Fig. 7. Retrieved scaling factor to the haze initial profile. The single retrievals are showed with different colors for different time of the season, as in the previous pictures. An average of the single haze retrieval in bins of $10^{\circ}$ of latitude is shown in black, with error bars. Note the hemispheric asymmetry in the haze, with the northern hemisphere being more opaque than the south during the northern winter season, as discussed in the text.

Despite these ambiguities the results on the haze appear quite reasonable in shape and range of variation: a hemispheric asymmetry in the haze is visible, with the northern hemisphere being about $40 \%$ more opaque during the northern winter season than the far south. In the northern hemisphere, around $40^{\circ}$ of latitude, the haze presents its maximum (single results for the 3 time periods and their average in bins are shown in Fig. 7). These results are in agreement with previous findings: Penteado and Griffith (2010) and Rannou et al. (2010). Vinatier et al. (2010), found a similar trend of latitudinal variation of the aerosol optical depth at 0.5 and 1.7 mbar in the stratosphere.

Very few data (not shown in the picture) indicated for the haze a scaling factor value around $2.5 / 3$, from $-60^{\circ}$ to $+60^{\circ}$ of latitude and present only in the night data of the first time period considered. These results were excluded for the moment because of possible calibration uncertainties (due to the number and type of deep space spectra used to calibrate the data). If the increased opacity is real, for example for the presence of clouds, a more sophisticated model will be required to correctly interpret the data.

Variations of the haze during the northern winter season are not clearly appreciable, except for the presence of more data with higher haze values in the second period (second part of the winter) in the north.

\subsection{Diurnal variations of surface temperature}

New and important information on diurnal variations of Titan surface temperature comes from the detection of an increase in temperature of about $1-1.5 \mathrm{~K}$ from the morning to the late afternoon.

The changing solar flux absorbed by the surface drives the seasonal, latitudinal and longitudinal (diurnal) variations of the surface temperature, but their amplitude depends highly on the surface properties, including the thermal inertia, albedo, composition and other physical properties.

Fits to our retrieved diurnal variations results using thermal models have been attempted in order to obtain more accurate constraints for surface properties, but the difficulty of taking into account in a thermal model all the atmospheric and surface 
a

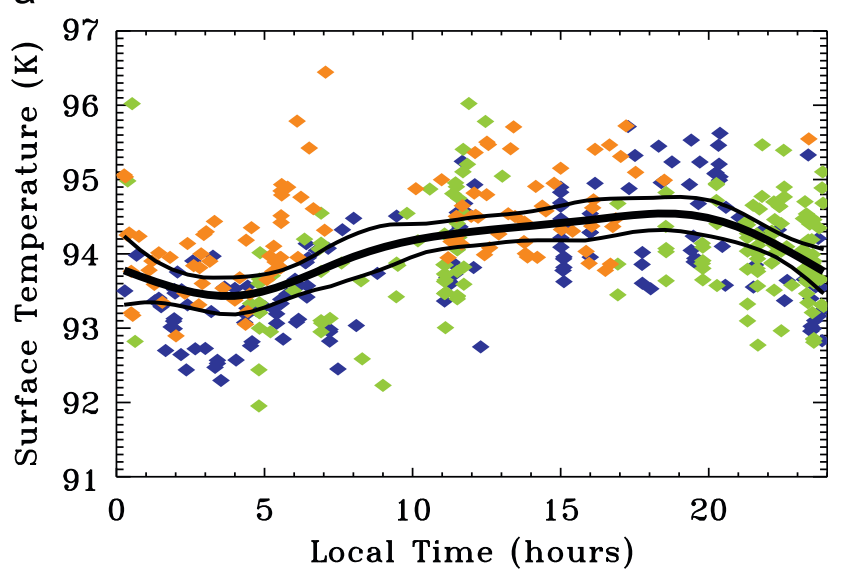

b

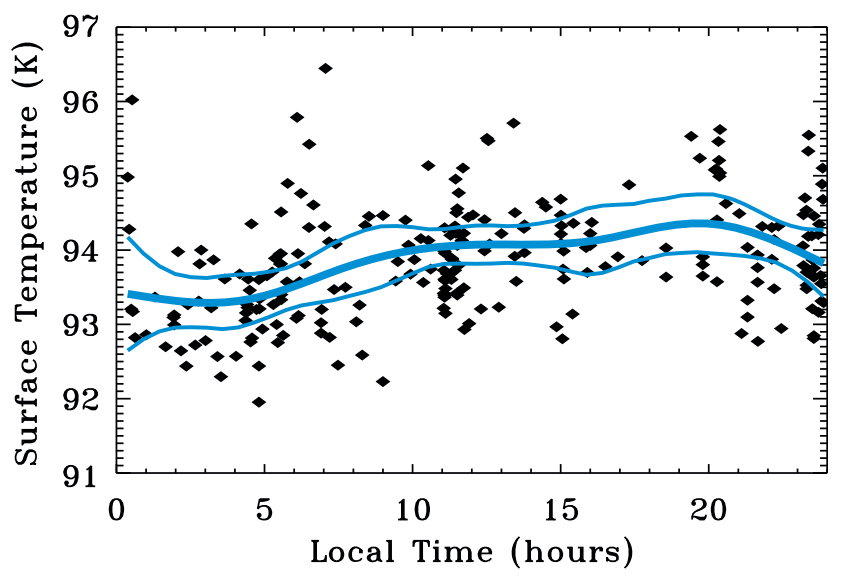

$\mathrm{C}$

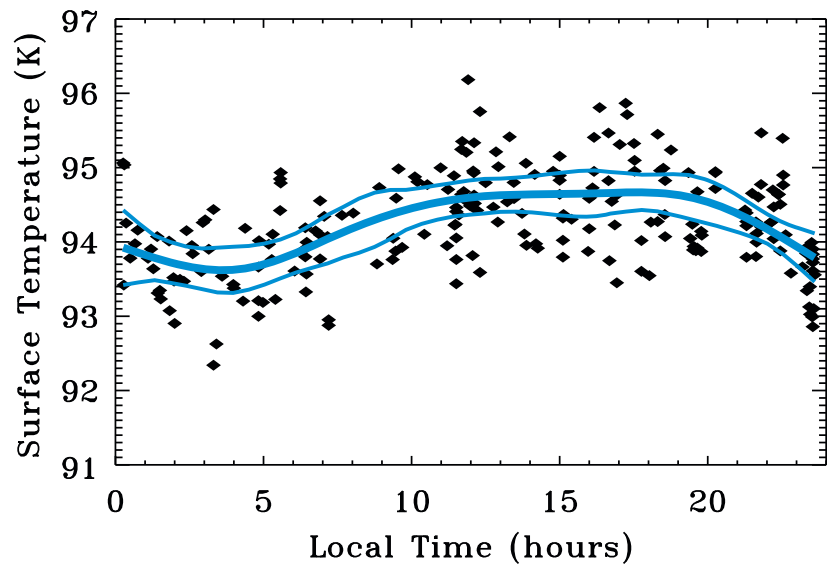

Fig. 8. Scatter plot of the surface temperature single retrievals versus Titan loca time for observations: (a) of $\pm 20^{\circ}$ of latitude around the Equator; (b) between 0 and $20^{\circ}$ of latitude; (c) between $0^{\circ}$ and $-20^{\circ}$ of latitude. In (a) the single retrieval are displayed in different colors from the 3 time periods (2004-2006 in blue, 2006-2008 in green, 2008-2010 in orange). In solid lines are shown the spline fit to the data and the 3-sigma fit errors. The shape of the variation is characterized by an increase in surface temperature starting at the beginning of the morning reaching a maximum of temperature varying between early and late afternoon, followed by a faster decrease during the night from around 20:00 to sunrise. In the southern hemisphere (c) the maximum around midday is more pronounced than in the northern hemisphere (b) where instead it seems to occur later in the afternoon.

processes involved in the surface temperature determination, including their spatial variations (for example different materials/ albedo/ inertia of different zones on the surface or lake

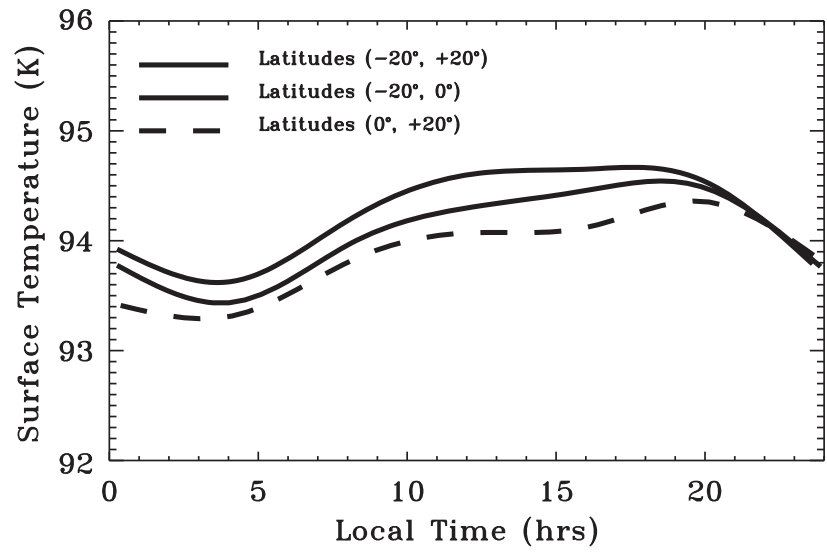

Fig. 9. Fit to the surface temperature retrievals versus Titan local time for observations: (a) of $\pm 20^{\circ}$ of latitude around the Equator (solid line); (b) $20^{\circ}$ of latitude north (dash line); (c) $20^{\circ}$ of latitude south (dash-dots line), presented in Fig. $8 \mathrm{a}-\mathrm{c}$ and shown here together. The highest values are associated with the observations of the southern part, in agreement with the subsolar point being below the equator during the northern winter/southern summer.

evaporation, some of these parameters not yet well known) makes it not possible at the moment to obtain quantitative results. To avoid including latitude dependence, in Fig. 8a we show the retrieved values of surface temperature as a function of local time for the region of $\pm 20^{\circ}$ around the equator.

The shape of the variation is distinctive: it shows an expected increase in surface temperature starting at the beginning of the morning, reaching then a maximum of temperature varying between early and late afternoon, followed by a faster decrease during the night from around 20:00 to sunrise. The amplitude of this diurnal variation was predicted for some surface types in the model of Tokano (2005): in Figs. 8 and 9 they show an example of the longitudinal variation of ground temperature, which is equivalent to the diurnal variation since the model is relative to a fixed time and a uniform surface. However, from the models of Tokano et al. (2005), of seasonal and diurnal changes, it was possible only to associate the diurnal changes of temperature obtained from our study with an average surface thermal inertia between 300 and $600 \mathrm{~J} \mathrm{~m}^{-2} \mathrm{~s}^{-1 / 2} \mathrm{~K}^{-1}$ and an albedo of 0.3 , in agreement mostly with porous icy regolith material.

The broad flat maximum in surface temperatures shown in Fig. 8 is curious. It is well known (Priestley, 1959; Gierasch and Goody, 1968) from modeling of terrestrial and Martian boundary layers that the surface temperature lags the solar heating, because of the effects of the surface thermal inertia. When the Sun is highest, much of the excess heat absorbed is conducted into the deeper sub-layers, as well as being radiated into the overlying atmosphere. Later in the day, as the subsurface warms up, the downward heat flux diminishes, and the surface temperatures increase. However, the terrestrial and Martian models indicate a surface temperature maximum no later than mid afternoon, whereas Fig. 8 indicates temperatures that do not decrease until 19:00 h local time. This may be related to Titan's optically thicker atmosphere and relatively greater backwarming in the thermal infrared, but the explanation for the broad maximum indicated in Fig. 8 is not completely understood.

In Fig. $8 \mathrm{~b}$ and $\mathrm{c}$ we show the results separated for the two hemispheres: from the equator to $20^{\circ}$ above and then the $20^{\circ}$ below the equator. We notice that in the southern hemisphere the maximum around mid-day is more pronounced than in the northern hemisphere where instead it seems to occur later in the afternoon. This later maximum remains particularly important in the northern hemisphere, where the increase due to the 
a
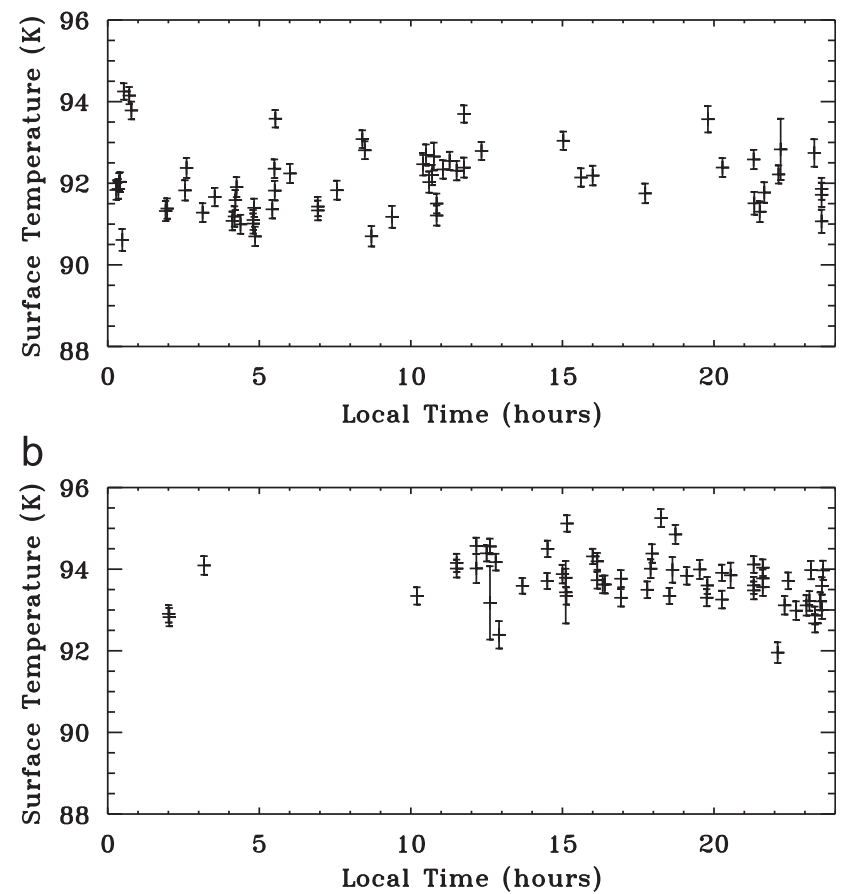

C
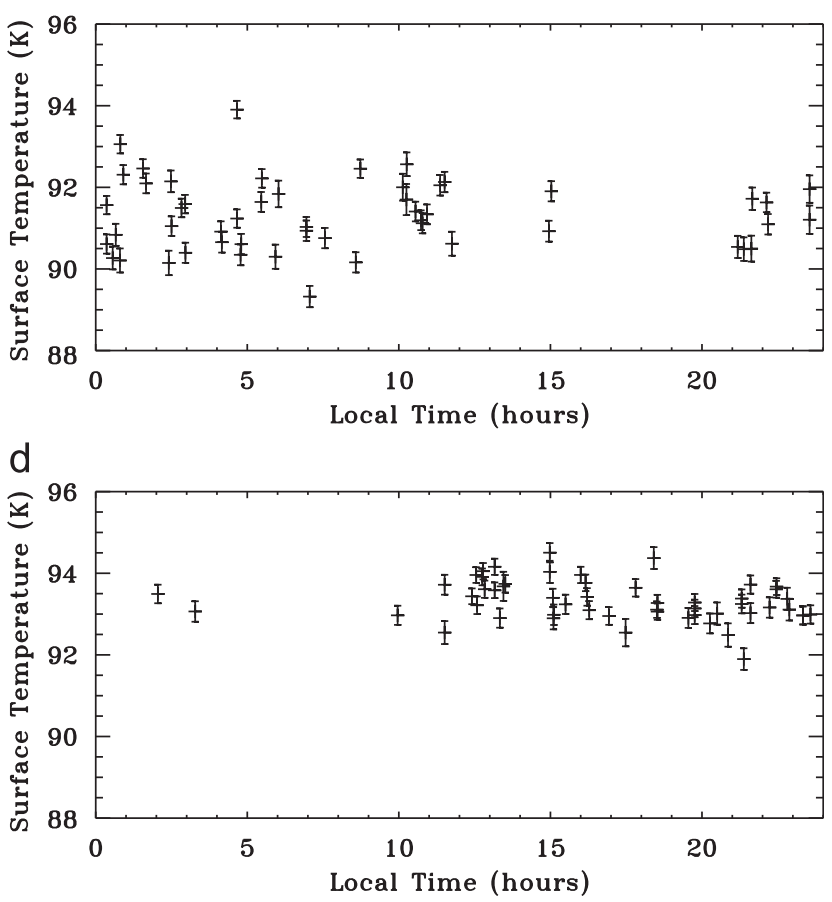

Fig. 10. Surface temperature results shown in sets of $10^{\circ}$ of latitude from $20^{\circ}$ to $40^{\circ}$. Unfortunately for higher latitudes than $20^{\circ}$ we may cover only a part of the day; however we see some evidence that the diurnal features are decreasing and then disappearing at latitudes greater than $20-30^{\circ}$ in the north and $30-40^{\circ}$ in the south due to the different insolation.

insolation becomes less efficient, due to the northern winter season positioning the subsolar point in the southern hemisphere.

In Fig. 9 the three fits to the surface temperature data versus local time of Fig. 8a-c are presented together. The highest values are associated with the observations of the southern $20^{\circ}$ from the equator, in agreement with the subsolar point being below the equator during the northern winter/southern summer, confirming the solidity of the obtained results.
We also separated the results in sets of $10^{\circ}$ of latitude to check if the diurnal effect was decreasing at higher latitudes due to the different insolation. Unfortunately there are not enough data that span the entire day in a bin of $10-20^{\circ}$ of latitude higher than $30^{\circ}$, since more than one third of the observations fall inside $\pm 20^{\circ}$ of latitude from the equator. Therefore for higher latitudes we may cover only a part of the day, so it is difficult to completely rule out the presence of a diurnal trend. However we see some evidence that the diurnal features are disappearing at latitudes greater than $20-30^{\circ}$ in the north and $30-40^{\circ}$ in the south (Fig. 10).

Due to a limited amount of data, it was not possible to subdivide the data used for the diurnal changes study, already selected in bins of latitude, in different time periods of the examined Titan season, since the coverage in local time would again be incomplete. However, to confirm the diurnal trend discovered in this work and rule out a bias with seasonal changes we display in Fig. 8a the three periods in different colors. We observe that the data, independently from the period of acquisition, fit well into the observed main diurnal trend of lower temperatures during the night with an increase starting from the early morning to the late afternoon. The data acquired during the vernal equinox, at the beginning of the northern spring, (orange color in Fig. 8a) contribute to the higher surface temperature values from midnight to early afternoon, with many data in the last part of the night and early morning up to $7 \mathrm{am}$, which might introduce an underestimation of the diurnal variation of about $0.5 \mathrm{~K}$, raising the range of surface temperature differences between night and day to $1.5 \mathrm{~K}$.

Since the diurnal range of the variation in temperature is small in absolute value, we searched for correlations of temperature with other parameters. To perform this check (besides a simple observation of scatter plots of the varying parameters) we computed the Pearson correlation coefficients (defined as the covariance of the two variables divided by the product of their standard deviations) of a few pairs of parameters. A very small correlation (less than 0.15) was found between the surface temperature and the emission angle, haze and satellite distance from the planet. Higher correlation coefficients were found with the latitude $(\sim 0.6)$, and with local time of low latitude observations $(\sim 0.4)$. An analysis of the emission angle versus the local time for all the data and for the three latitude bins considered in Fig. 8 showed no correlation between the two parameters.

In addition, we tried to remove possible bias in the results related to the higher albedo in the Xanadu region. We reanalyzed the surface temperature excluding this area; only a few data around 11:00 in the morning were excluded and the shape of the diurnal variations was not significantly affected. If more data become available over this and other regions characterized by interesting and diverse albedo features, a correlation of the surface temperature with the albedo can be investigated; for the moment in this study eventual differences in surface temperature due to differences in surface properties (e.g. albedo) result in a scatter of the above described surface temperature results, without anyway influencing the general trends observed in latitude and time.

\section{Conclusions and future improvements}

For the first time this work has made it possible to discern the existence of diurnal variations in the surface temperature of Titan. Such a possibility has been previously discussed and expected by some models (Tokano et al., 2005), but there has not previously been confirmation.

The retrieved diurnal variations of about $1.5 \mathrm{~K}$ are a further constraint for Titan models, while the latitudinal dependence 
found in this work has provided validation of previous results from Voyager and Cassini.

As soon as better knowledge of the Titan tropospheric temperature profile dependence at higher latitudes is available, we will be able to provide reliable temperature estimates in the polar regions. Furthermore, after further flybys and new acquisitions it will be possible to improve the surface temperature map coverage and possibly give some additional constraints on the surface properties. A longer period of observations, being the Cassini mission extended to 2017, will lead to a better subdivision in time of the data and consequently an improved retrieval of seasonal variations.

\section{Acknowledgments}

The authors acknowledge and thank the efforts of the Cassini CIRS operations team that made possible this study of Titan data. Authors Cottini and Nixon thank the continued support of the Cassini Data Analysis Program (CDAP) Grant number NNX08AP74G during the research and writing of this paper. We also wish to thank Nicolas Gorius for discussions and help with improving the data calibrations, and Tetsuya Tokano, Robert Samuelson and Carrie Anderson for interesting discussions regarding the implications of this work.

\section{References}

Borysow, A., 1991. Modelling of collision-induced infrared-absorption spectra of $\mathrm{H}_{2}-\mathrm{H}_{2}$ pairs in the fundamental band at temperatures from $20 \mathrm{~K}$ to $300 \mathrm{~K}$. Icarus 92 (2), 273-279.

Borysow, A., Frommhold, L., 1986a. Theoretical collision-induced rototranslationa absorption spectra for modeling Titan's atmosphere $-\mathrm{H}_{2}-\mathrm{N}_{2}$ pairs. Astrophys. J. 303, 495-510.

Borysow, A., Frommhold, L., 1986b. Theoretical collision-induced rototranslational absorption spectra for the outer planets $-\mathrm{H}_{2}-\mathrm{CH}_{4}$ pairs. Astrophys. J. 304 849-865.

Borysow, A., Frommhold, L., 1986c. Collision-induced rototranslational absorption spectra of $\mathrm{N}_{2}-\mathrm{N}_{2}$ pairs for temperatures from 50 to $300 \mathrm{~K}$. Astrophys. J. 311, 1043-1057.

Borysow, A., Frommhold, L., 1987. Collision-induced rototranslational absorption spectra of $\mathrm{CH}_{4}-\mathrm{CH}_{4}$ pairs at temperatures from 50 to $300 \mathrm{~K}$. Astrophys. J. 318, 940-943.

Borysow, A., Tang, C., 1993. Far infrared CIA spectra of $\mathrm{N}_{2}-\mathrm{CH}_{4}$ pairs for modelling of Titan's atmosphere. Icarus 105, 175-183.

Courtin, R.D., Gautier, D., McKay, C.P., 1995. Titan's thermal emission spectrum: reanalysis of the voyager infrared measurements. Icarus 114 (1), 144-162.

Courtin, R.D., Sim, C., Kim, S., Gautier, D., Jennings, D.E., 2008. Latitudinal variations of tropospheric $\mathrm{H}_{2}$ on Titan from the Cassini CIRS investigation. Bull. Am. Astron. Soc. 40, 446. de Kok, R., Irwin, P.G.J., Teanby, N.A., Nixon, C.A., Jennings, D.E., Fletcher, L., Howett, C., Calcutt, S.B., Bowles, N.E., Flasar, F.M., Taylor, F.W., 2007. Characteristics of Titan's stratospheric aerosols and condensate clouds from Cassini CIRS far-infrared spectra. Icarus 191, 223-235.

de Kok, R., Irwin, P.G.J., Teanby, N.A., 2010. Far-infrared opacity sources in Titan's troposphere reconsidered. Icarus 209 (2), 854-857.

Flasar, F.M., Samuelson, R.E., Conrath, B.J., 1981. Titan's atmosphere: temperature and dynamic. Nature 292, 693.

Flasar, F.M., et al., 2004. Exploring the Saturn system in the thermal infrared: the composite infrared spectrometer. Space Sci. Rev. 115, 169-297.

Fulchignoni, M., et al., 2005. In situ measurements of the physical characteristics of Titan's environment. Nature 438, 785.

Gierasch, P., Goody, R., 1968. A study of the thermal and dynamical structure of the Martian lower atmosphere. Planet. Space Sci. 16, 615-646.

Hanel, R.A., et al., 1981. Infrared observations of the Saturnian system from Voyager 1. Science 212, 192

Irwin, P.G.J., Teanby, N.A., de Kok, R., Fletcher, L.N., Howett, C.J.A., Tsang, C.C.C., Wilson, C.F., Calcutt, S.B., Nixon, C.A., Parrish, P.D., 2008. The NEMESIS planetary atmosphere radiative transfer and retrieval tool. J. Quant. Spectrosc Radiat. Transf. 109, 1136-1150.

Jennings, D.E., et al., 2009. Titan's surface brightness temperatures. The Astrophysical Journal Letters 691 (2), L103-L105.

Lacis, A.A., Oinas, V., 1991. A description of the correlated $\mathrm{k}$ distributionmethod for modelling nongray gaseous absorption, thermal emission, andmultiple-scattering in vertically inhomogeneous atmospheres. J. Geophys. Res. 96 (D5), 9027-9063.

Niemann, H.B., Atreya, S.K., Bauer, S.J., Carignan, G.R., Demick, J.E., Frost, R.L Gautier, D., Haberman, J.A., Harpold, D.N., Hunten, D.M., Israel, G., Lunine, J.I., Kasprzak, W.T., Owen, T.C., Paulkovich, M., Raulin, F., Raaen, E., Way, S.H., 2005. The abundances of constituents of Titan's atmosphere from the GCMS instrument on the Huygens probe: Huygens probe on titan. Nature 438 (7069) 779-784.

Penteado, P.F., Griffith, C.A., 2010. Ground-based measurements of the methane distribution on Titan. Icarus 206 (1), 345-351.

Penteado, P.F., Griffith, C.A., Tomasko, M.G., Engel, S., See, C., Doose, L., Baines, K.H., Brown, R.H., Buratti, B.J., Clark, R., Nicholson, P., Sotin, C., 2010. Latitudinal variations in Titan's methane and haze from Cassini VIMS observations. Icarus 206, 352-365.

Priestley, C.H.B., 1959. Turbulent Transfer in the Lower Atmosphere. University of Chicago Press Ch. 8.

Rannou, P., Cours, T., Le Mouélic, S., Rodriguez, S., Sotin, C., Drossart, P., Brown, R., 2010. Titan haze distribution and optical properties retrieved from recent observations. Icarus 208 (2), 850-867.

Rodgers, C.D., 2000. Inverse Methods for Atmospheric Sounding: Theory and Practice. World Scientific, Singapore.

Rothman, L.S., et al., 2005. The HITRAN 2004 molecular spectroscopic database. J. Quant. Spectrosc. Radiat. Trans. 96, 139-204.

Samuelson, R.E., Hanel, R.A., Kunde, V.G., Maguire, W.C., 1981. The mean molecular weight and hydrogen abundance of Titan's atmosphere. Nature 292, 688.

Teanby, N.A., 2007. Constrained Smoothing of noisy data using splines in tension. Math. Geol. 39 (4), 419-434.

Tokano, T., 2005. Meteorological assessment of the surface temperatures on Titan: constraints on the surface type. Icarus 173, 222-242.

Vinatier, S., Bézard, B., de Kok, R., Anderson, C.M., Samuelson, R.E., Nixon, C.A., Mamoutkine, A., Carlson, R.C., Jennings, D.E., Guandique, E.A., Bjoraker, G.L., Flasar, F.M., Kunde, V.G., 2010. Analysis of Cassini/CIRS limb spectra of Titan acquired during the nominal mission. II: aerosol extinction profiles in the $600-1420 \mathrm{~cm}^{-1}$ spectral range. Icarus 210 (2), 852-866. 